\title{
CRITERION FOR LOGARITHMIC CONNECTIONS WITH PRESCRIBED RESIDUES
}

\author{
INDRANIL BISWAS, ANANYO DAN, AND ARJUN PAUL
}

\begin{abstract}
A theorem of Weil and Atiyah says that a holomorphic vector bundle $E$ on a compact Riemann surface $X$ admits a holomorphic connection if and only if the degree of every direct summand of $E$ is zero. Fix a finite subset $S$ of $X$, and fix an endomorphism $A(x) \in \operatorname{End}\left(E_{x}\right)$ for every $x \in S$. It is natural to ask when there is a logarithmic connection on $E$ singular over $S$ with residue $A(x)$ at every $x \in S$. We give a necessary and sufficient condition for it under the assumption that the residues $A(x)$ are rigid.
\end{abstract}

\section{INTRODUCTION}

Let $X$ be a compact connected Riemann surface. Let $E$ be a holomorphic vector bundle on $X$. A holomorphic connection on $E$ is a flat connection on $E$ such that the (locally defined) flat sections of $E$ are holomorphic. It is known that $E$ admits a holomorphic connection if and only if every direct summand of $E$ is of degree zero [We], [At2]. In particular, an indecomposable holomorphic vector bundle on $X$ admits a holomorphic connection if and only if its degree is zero.

Fix a finite subset $S \subset X$. For each point $x \in S$, fix an endomorphism $A(x) \in$ $\operatorname{End}\left(E_{x}\right)$ of the fiber $E_{x}$ of the vector bundle $E$. Here we address the following question: When there is a logarithmic connection on $E$ singular over $S$ with residue $A(x)$ at every $x \in S$ ?

First take the case where $E$ is simple, meaning all global holomorphic endomorphisms of $E$ are scalar multiplications. We prove the following (see Lemma 3.2):

Lemma 1.1. Let $E$ be a simple holomorphic vector bundle on $X$. Then the following two statements are equivalent:

(1) There is a logarithmic connection on $E$ singular over $S$ with residue $A(x)$ for every $x \in S$.

(2) The collection of endomorphisms $A(x), x \in S$, satisfy the condition

$$
\operatorname{degree}(E)+\sum_{x \in S} \operatorname{trace}(A(x))=0 .
$$

Note that Lemma 1.1 holds for stable vector bundles because they are simple.

2010 Mathematics Subject Classification. 53B15, 14H60.

Key words and phrases. Logarithmic connection, residue, rigidity, logarithmic Atiyah bundle. 
An endomorphism $A \in \operatorname{End}\left(E_{x}\right)$, where $x \in X$, will be called rigid if $A$ commutes with every global holomorphic endomorphism of $E$. In other words, $A$ is rigid if and only if $A \circ v(x)=v(x) \circ A$ for all $v \in H^{0}(X, \operatorname{End}(E))$.

Now take the case where the holomorphic vector bundle $E$ is indecomposable, meaning it does not split into a direct sum of holomorphic vector bundles of positive ranks. We prove the following (see Proposition 4.1):

Proposition 1.2. Let $E$ be an indecomposable vector bundle on $X$. For each point $x \in S$, fix a rigid endomorphism $A(x) \in \operatorname{End}\left(E_{x}\right)$. Then the following are equivalent:

(1) There is a logarithmic connection on $E$ singular over $S$ such that the residue is $A(x)$ for every $x \in S$.

(2) The collection $A(x), x \in S$, satisfy the condition

$$
\operatorname{degree}(E)+\sum_{x \in S} \operatorname{trace}(A(x))=0 .
$$

Using Proposition 1.2, the following is proved (see Theorem 4.2):

Theorem 1.3. Let $E$ be a holomorphic vector bundle on $X$. For each point $x \in S$, fix a rigid endomorphism $A(x) \in \operatorname{End}\left(E_{x}\right)$. Then the following hold:

(1) For every direct summand $F \subset E$,

$$
A(x)\left(F_{x}\right) \subset F_{x}
$$

for every $x \in S$.

(2) The vector bundle $E$ admits a logarithmic connection on $E$ singular over $S$, with residue $A(x)$ at every $x \in S$, if and only if for every direct summand $F \subset E$,

$$
\operatorname{degree}(F)+\sum_{x \in S} \operatorname{trace}\left(\left.A(x)\right|_{F_{x}}\right)=0 .
$$

\section{LOGARITHMIC CONNECTIONS AND RESIDUE}

2.1. Logarithmic connections on $E$. Let $X$ be a compact connected Riemann surface. Let $E$ be a holomorphic vector bundle on $X$. The fiber of $E$ over any point $x \in X$ will be denoted by $E_{x}$.

Fix finitely many distinct points

$$
S:=\left\{x_{1}, \cdots, x_{d}\right\} \subset X .
$$

The reduced effective divisor $x_{1}+\ldots+x_{d}$ on $X$ will also be denoted by $S$.

The holomorphic cotangent bundle $\Omega_{X}$ of $X$ will also be denoted by $K_{X}$. A logarithmic connection on $E$ singular over $S$ is a first order holomorphic differential operator

$$
D: E \longrightarrow E \otimes \Omega_{X}(\log S)=E \otimes K_{X} \otimes \mathcal{O}_{X}(S)
$$

satisfying the Leibniz identity which says that

$$
D(f s)=f D(s)+s \otimes(d f),
$$


where $s$ is any locally defined holomorphic section of $E$ and $f$ is any locally defined holomorphic function on $X$ [De].

For any point $x \in S$, the fiber $\left(K_{X} \otimes \mathcal{O}_{X}(S)\right)_{x}$ is identified with $\mathbb{C}$ using the Poincaré adjunction formula [GH, p. 146]. More explicitly, for any holomorphic coordinate $z$ around $x$ with $z(x)=0$, the image of $\frac{d z}{z}$ in $\left(K_{X} \otimes \mathcal{O}_{X}(S)\right)_{x}$ is independent of the choice of the coordinate function; the above identification between $\left(K_{X} \otimes \mathcal{O}_{X}(S)\right)_{x}$ and $\mathbb{C}$ sends this independent image to $1 \in \mathbb{C}$. For a logarithmic connection $D$ on $E$ singular over $S$, and any $x \in S$, consider the composition

$$
E \stackrel{D}{\longrightarrow} E \otimes K_{X} \otimes \mathcal{O}_{X}(S) \longrightarrow E_{x} \otimes\left(K_{X} \otimes \mathcal{O}_{X}(S)\right)_{x}=E_{x} .
$$

The above Leibniz identity implies that it is $\mathcal{O}_{X}$-linear; hence this composition is an element of $\operatorname{End}\left(E_{x}\right)=\operatorname{End}(E)_{x}$. This element of $\operatorname{End}\left(E_{x}\right)$, which we will denote by $\operatorname{Res}(D, x)$, is called the residue of $D$ at $x$ [De, p. 53].

Let $\operatorname{Diff}^{i}(E, E)$ be the holomorphic vector bundle on $X$ whose holomorphic sections over any open subset $U \subset X$ are the holomorphic differential operators $\left.\left.E\right|_{U} \longrightarrow E\right|_{U}$ of order at most $i$. So we have a short exact sequence of holomorphic vector bundles

$$
0 \longrightarrow \operatorname{End}(E)=\operatorname{Diff}^{0}(E, E) \longrightarrow \operatorname{Diff}^{1}(E, E) \stackrel{\sigma}{\longrightarrow} \operatorname{End}(E) \otimes T X \longrightarrow 0,
$$

where $T X$ is the holomorphic tangent bundle of $X$, and $\sigma$ is the symbol homomorphism. Now define the subsheaf

$$
\operatorname{At}(E)(-\log S):=\sigma^{-1}\left(\operatorname{Id}_{E} \otimes_{\mathbb{C}} T X \otimes \mathcal{O}_{X}(-S)\right) \subset \operatorname{Diff}^{1}(E, E) .
$$

It fits in the following short exact sequence of holomorphic vector bundles on $X$ obtained from (2.1):

$$
0 \longrightarrow \operatorname{End}(E) \longrightarrow \operatorname{At}(E)(-\log S) \stackrel{\widehat{\sigma}}{\longrightarrow} T X(-\log S):=T X \otimes \mathcal{O}_{X}(-S) \longrightarrow 0,
$$

where $\widehat{\sigma}$ is the restriction of $\sigma$ to the subsheaf $\operatorname{At}(E)(-\log S)$. A logarithmic connection on $E$ singular over $S$ is a holomorphic splitting of (2.2), meaning a $\mathcal{O}_{X}$-linear homomorphism

$$
h: T X(-\log S) \longrightarrow \operatorname{At}(E)(-\log S)
$$

such that $\widehat{\sigma} \circ h=\operatorname{Id}_{T X(-\log S)}$.

For any $x \in S$, the composition

$$
\operatorname{At}(E)(-\log S)_{x} \longrightarrow \operatorname{Diff}^{1}(E, E)_{x} \stackrel{\sigma(x)}{\longrightarrow}(\operatorname{End}(E) \otimes T X)_{x}
$$

vanishes; the above homomorphism $\operatorname{At}(E)(-\log S)_{x} \longrightarrow \operatorname{Diff}^{1}(E, E)_{x}$ is given by the inclusion of $\operatorname{At}(E)(-\log S)$ in $\operatorname{Diff}^{1}(E, E)$. Therefore, from (2.1) we get a homomorphism

$$
j_{x}: \operatorname{At}(E)(-\log S)_{x} \longrightarrow \operatorname{End}\left(E_{x}\right)=\operatorname{End}(E)_{x} .
$$

For any logarithmic connection $h$ as in (2.3), the element

$$
j_{x}(h(x)(1)) \in \operatorname{End}\left(E_{x}\right)
$$

is the residue of $h$ at $x$; here $1 \in \mathbb{C}$ is considered as an element of $T X(-\log S)_{x}$ using the earlier mentioned identification between $T X(-\log S)_{x}$ and $\mathbb{C}$. 


\subsection{Logarithmic connections with given residues.}

Lemma 2.1. For any $x \in S$, the fiber $\operatorname{At}(E)(-\log S)_{x}$ has a canonical decomposition

$$
\operatorname{At}(E)(-\log S)_{x}=\operatorname{End}(E)_{x} \oplus T X(-\log S)_{x}=\operatorname{End}\left(E_{x}\right) \oplus \mathbb{C} .
$$

Proof. We recall the definition of the Atiyah bundle:

$$
\operatorname{At}(E):=\sigma^{-1}\left(\operatorname{Id}_{E} \otimes_{\mathbb{C}} T X\right) \subset \operatorname{Diff}^{1}(E, E),
$$

where $\sigma$ is the symbol map in (2.1) [At2]. It fits in the Atiyah exact sequence

$$
0 \longrightarrow \operatorname{End}(E) \longrightarrow \operatorname{At}(E) \stackrel{\widetilde{\sigma}}{\longrightarrow} T X \longrightarrow 0,
$$

where $\widetilde{\sigma}$ is the restriction of $\sigma$. Let

$$
0 \longrightarrow \operatorname{End}(E) \otimes \mathcal{O}_{X}(-S) \longrightarrow \operatorname{At}(E) \otimes \mathcal{O}_{X}(-S) \longrightarrow T X \otimes \mathcal{O}_{X}(-S) \longrightarrow 0
$$

be the tensor product of the Atiyah exact sequence with the line bundle $\mathcal{O}_{X}(-S)$. From this exact sequence and (2.2) it follows immediately that

$$
\operatorname{At}(E) \otimes \mathcal{O}_{X}(-S) \subset \operatorname{At}(E)(-\log S) .
$$

For any $x \in S$, from this inclusion of coherent sheaves and the exact sequences (2.2) and (2.5) we have the commutative diagram

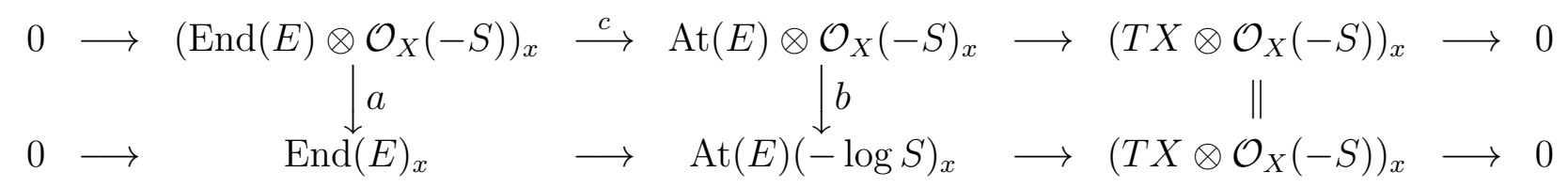

where $a$ is the zero homomorphism because $x \in S$. Now from the snake lemma, [La, p. 158, Lemma 9.1], it follows that the kernel of the homomorphism $b$ coincides with the image of $c$. Hence the image of the fiber $\left(\operatorname{At}(E) \otimes \mathcal{O}_{X}(-S)\right)_{x}$ in $\operatorname{At}(E)(-\log S)_{x}$ is identified with the quotient line $T X(-\log S)_{x}=\mathbb{C}$ of $\operatorname{At}(E) \otimes \mathcal{O}_{X}(-S)_{x}$. It is easy to see that this image $T X(-\log S)_{x} \subset \operatorname{At}(E)(-\log S)_{x}$ of $b$ coincides with the kernel of the homomorphism $j_{x}$ in (2.4). On the other hand, from (2.2) it follows that $\operatorname{End}(E)_{x} \subset$ $\operatorname{At}(E)(-\log S)_{x}$. Now it is straight-forward to check that the resulting homomorphism

$$
\operatorname{End}(E)_{x} \oplus T X(-\log S)_{x} \longrightarrow \operatorname{At}(E)(-\log S)_{x}
$$

is an isomorphism.

For each point $x \in S$, fix an endomorphism

$$
A(x) \in \operatorname{End}\left(E_{x}\right) .
$$

In view of Lemma 2.1, we have the complex line

$$
\ell_{x}:=\mathbb{C} \cdot(A(x), 1) \subset \operatorname{End}\left(E_{x}\right) \oplus \mathbb{C}=\operatorname{At}(E)(-\log S)_{x} .
$$

Let $\mathcal{A}(E) \longrightarrow X$ be the holomorphic vector bundle that fits in the short exact sequence of coherent sheaves

$$
0 \longrightarrow \mathcal{A}(E) \longrightarrow \operatorname{At}(E)(-\log S) \longrightarrow \bigoplus_{x \in S} \operatorname{At}(E)(-\log S)_{x} / \ell_{x} \longrightarrow 0
$$


where $\ell_{x}$ is the line constructed above. From (2.2) we have the short exact sequence

$$
0 \longrightarrow \operatorname{End}(E) \otimes \mathcal{O}_{X}(-S) \longrightarrow \mathcal{A}(E) \stackrel{\sigma^{\prime}}{\longrightarrow} T X(-\log S) \longrightarrow 0
$$

where $\sigma^{\prime}$ is the restriction of the homomorphism $\widehat{\sigma}$ in (2.2).

The following lemma is a straight-forward consequence of the definitions of logarithmic connection and residue:

Lemma 2.2. A logarithmic connection on $E$ singular over $S$ with residue $A(x), x \in S$, is a holomorphic homomorphism

$$
h: T X(-\log S) \longrightarrow \mathcal{A}(E)
$$

such that $\sigma^{\prime} \circ h=\operatorname{Id}_{T X(-\log S)}$, where $\sigma^{\prime}$ is the homomorphism in (2.7).

Lemma 2.3. For each point $x \in S$, fix a number $\lambda_{x} \in \mathbb{C}$. Let $L$ be a holomorphic line bundle on $X$. There is a logarithmic connection on $L$ singular over $S$ with residue $\lambda_{x}$ at each $x \in S$ if and only if

$$
\operatorname{degree}(L)+\sum_{x \in S} \lambda_{x}=0
$$

Proof. If $D$ is a logarithmic connection on $L$ of the above type, then from $[\mathrm{Oh}, \mathrm{p} .16$, Theorem 3] we know that (2.8) holds.

To prove the converse, assume that (2.8) holds. Fix a point $x_{0} \in X \backslash S$. Since the two holomorphic line bundles $L$ and $L_{0}:=\mathcal{O}_{X}\left(\operatorname{degree}(L) \cdot x_{0}\right)$ differ by tensoring with a holomorphic line bundle of degree zero, and any holomorphic line bundle of degree zero on $X$ admits a nonsingular holomorphic connection (this follows immediately from the Atiyah-Weil criterion), it suffices to show that $L_{0}$ admits a logarithmic connection singular over $S$ with residue $\lambda_{x}$ at each $x \in S$.

For the exact sequence of coherent sheaves

$$
0 \longrightarrow K_{X} \longrightarrow K_{X} \otimes \mathcal{O}_{X}\left(S+x_{0}\right) \longrightarrow \bigoplus_{y \in S \cup\left\{x_{0}\right\}} \mathbb{C}_{y} \longrightarrow 0
$$

where $\mathbb{C}_{y}$ is a copy of $\mathbb{C}$ supported at $y$, let

$$
H^{0}\left(X, K_{X} \otimes \mathcal{O}_{X}\left(S+x_{0}\right)\right) \longrightarrow \bigoplus_{y \in S \cup\left\{x_{0}\right\}} \mathbb{C}_{y} \stackrel{\xi}{\longrightarrow} H^{1}\left(X, K_{X}\right)=\mathbb{C}
$$

be the exact sequence of cohomologies. The above homomorphism $\xi$ sends $\bigoplus_{y \in S \cup\left\{x_{0}\right\}} c_{y}$ to $\sum_{y \in S \cup\left\{x_{0}\right\}} c_{y}$. Therefore, from (2.8) it follows that there is a holomorphic section

$$
\omega_{0} \in H^{0}\left(X, K_{X} \otimes \mathcal{O}_{X}\left(S+x_{0}\right)\right)
$$

whose residue over any $x \in S$ is $\lambda_{x}$ while the residue over $x_{0}$ is degree $(L)=-\sum_{x \in S} \lambda_{x}$. Now the logarithmic connection $d+\omega_{0}$ on $\mathcal{O}_{X}\left(\operatorname{degree}(L) \cdot x_{0}\right)=L_{0}$, where $d$ is the usual de Rham differential, has the required residues. 


\section{The EXTEnsion Class}

Isomorphism classes of extensions of the line bundle $T X(-\log S)$ by the vector bundle $\operatorname{End}(E) \otimes \mathcal{O}_{X}(-S)$ are parametrized by

$$
H^{1}\left(X, \operatorname{Hom}\left(T X(-\log S), \operatorname{End}(E) \otimes \mathcal{O}_{X}(-S)\right)\right)=H^{1}\left(X, \operatorname{End}(E) \otimes K_{X}\right) .
$$

Consider the extension in (2.7). Let

$$
\phi_{E}^{A} \in H^{1}\left(X, \operatorname{Hom}\left(T X(-\log S), \operatorname{End}(E) \otimes \mathcal{O}_{X}(-S)\right)\right)=H^{1}\left(X, \operatorname{End}(E) \otimes K_{X}\right)
$$

be the corresponding cohomology class. Our aim in this section is to determine $\phi_{E}^{A}$.

Take any point $y \in X$. We will construct a homomorphism

$$
\gamma_{y}: \operatorname{End}(E)_{y} \longrightarrow H^{1}\left(X, \operatorname{End}(E) \otimes K_{X}\right) .
$$

For this, consider the short exact sequence of sheaves on $X$

$$
0 \longrightarrow \mathcal{O}_{X} \longrightarrow \mathcal{O}_{X}(y) \longrightarrow \mathcal{O}_{X}(y)_{y}=T_{y} X \longrightarrow 0
$$

(recall that the Poincaré adjunction formula identifies the fiber $\mathcal{O}_{X}(y)_{y}$ with the fiber $T_{y} X$ of the holomorphic tangent bundle). Tensoring this exact sequence with $\operatorname{End}(E) \otimes K_{X}$, the following short exact sequence is obtained

$$
0 \longrightarrow \operatorname{End}(E) \otimes K_{X} \longrightarrow \operatorname{End}(E) \otimes K_{X} \otimes \mathcal{O}_{X}(y) \longrightarrow \operatorname{End}(E)_{y} \longrightarrow 0
$$

The homomorphism $\gamma_{y}$ in (3.2) is the one occurring in the long exact sequence of cohomologies associated to the above short exact sequence.

Consider the Atiyah exact sequence in (2.5). Let

$$
\phi_{E}^{0} \in H^{1}(X, \operatorname{Hom}(T X, \operatorname{End}(E)))=H^{1}\left(X, \operatorname{End}(E) \otimes K_{X}\right)
$$

be the extension class for it.

Proposition 3.1. The cohomology class $\phi_{E}^{A}$ in (3.1) coincides with

$$
\phi_{E}^{0}+\sum_{x \in S} \gamma_{x}(A(x)) \in H^{1}\left(X, \operatorname{End}(E) \otimes K_{X}\right),
$$

where $\phi_{E}^{0}$ and $\gamma_{x}$ are constructed in (3.3) and (3.2) respectively.

Proof. We will give an alternative description of the homomorphism $\gamma_{y}$ in (3.2). First note that Serre duality says that

$$
H^{1}\left(X, \operatorname{End}(E) \otimes K_{X}\right)=H^{0}(X, \operatorname{End}(E))^{*} .
$$

Let

$$
\widetilde{\gamma}_{y}: \operatorname{End}(E)_{y} \longrightarrow H^{0}(X, \operatorname{End}(E))^{*}
$$

be the homomorphism produced by $\gamma_{y}$ using the above Serre duality. For any $\alpha \in$ $\operatorname{End}(E)_{y}$ and $\beta \in H^{0}(X, \operatorname{End}(E))$, it can be checked that

$$
\widetilde{\gamma}_{y}(\alpha)(\beta)=\operatorname{trace}(\alpha \circ(\beta(y))) \text {. }
$$

Next we recall a Dolbeault type description of $\phi_{E}^{0}$. Let

$$
\widetilde{\phi}_{E}^{0} \in H^{0}(X, \operatorname{End}(E))^{*}
$$


be the element corresponding to $\phi_{E}^{0}$ in (3.3) by the isomorphism in (3.4).

A $C^{\infty}$ homomorphism

$$
H: T X \longrightarrow \operatorname{At}(E)
$$

such that $\widetilde{\sigma} \circ H=\operatorname{Id}_{T X}$ (see $(2.5)$ for $\tilde{\sigma}$ ) defines a $C^{\infty}$ connection on $E$ whose $(0,1)$ component coincides with the Dolbeault operator on the holomorphic vector bundle $E$. Let $\mathcal{K}(H)$ be the curvature of the $C^{\infty}$ connection on $E$ given by $H$; it is a $(1,1)$-form with values in $\operatorname{End}(E)$. Therefore, $\mathcal{K}(H)$ represents an element of Dolbeault cohomology $H^{1}\left(X, \operatorname{End}(E) \otimes K_{X}\right)$. This element of $H^{1}\left(X, \operatorname{End}(E) \otimes K_{X}\right)$ represented by $\mathcal{K}(H)$ coincides with $\phi_{E}^{0}$ in (3.3). Consequently, for any $\beta \in H^{0}(X, \operatorname{End}(E))$, we have

$$
\widetilde{\phi}_{E}^{0}(\beta)=\int_{X} \operatorname{trace}(\mathcal{K}(H) \circ \beta),
$$

where $\widetilde{\phi}_{E}^{0}$ is defined in $(3.7)$.

The cohomology class $\phi_{E}^{A}$ has a similar description. Using (3.4), the cohomology class $\phi_{E}^{A}$ in (3.1) defines an element

$$
\widetilde{\phi}_{E}^{A} \in H^{0}(X, \operatorname{End}(E))^{*} .
$$

Let

$$
H^{\prime}: T X(-\log S) \longrightarrow \mathcal{A}(E)
$$

be a $C^{\infty}$ homomorphism such that

- $H^{\prime}$ is holomorphic around the points of $S$, and

- $\sigma^{\prime} \circ H^{\prime}=\operatorname{Id}_{T X(-\log S)}$, where $\sigma^{\prime}$ is the homomorphism in (2.7).

Let $\mathcal{K}\left(H^{\prime}\right)$ be the curvature of the singular $C^{\infty}$ connection on $E$ defined by $H^{\prime}$. We note that $\mathcal{K}\left(H^{\prime}\right)$ is a $C^{\infty}$ two-form on $X$ with values in $\operatorname{End}(E)$; this is because $\mathcal{K}\left(H^{\prime}\right)$ vanishes in a neighborhood of $S$, so it is an $\operatorname{End}(E)$ valued $C^{\infty}$ two-form on entire $X$. For any $\beta \in H^{0}(X, \operatorname{End}(E))$,

$$
\widetilde{\phi}_{E}^{A}(\beta)=\int_{X} \operatorname{trace}\left(\mathcal{K}\left(H^{\prime}\right) \circ \beta\right)
$$

The proposition is a straight-forward consequence of the above descriptions of $\gamma_{y}, \phi_{E}^{0}$ and $\phi_{E}^{A}$.

Lemma 3.2. Let $E$ be a simple vector bundle, meaning $H^{0}(X, \operatorname{End}(E))=\mathbb{C} \cdot \operatorname{Id}_{E}$. Then the following two statements are equivalent:

(1) There is a logarithmic connection on $E$ singular over $S$ with residue $A(x)$ for every $x \in S$.

(2) The collection of endomorphisms $A(x), x \in S$, satisfy the condition

$$
\operatorname{degree}(E)+\sum_{x \in S} \operatorname{trace}(A(x))=0 .
$$

Proof. If $E$ admits a logarithmic connection singular over $S$ such that residue is $A(x)$ for every $x \in S$, then degree $(E)+\sum_{x \in S} \operatorname{trace}(A(x))=0$ [Oh, p. 16, Theorem 3]. 
To prove the converse, assume that

$$
\operatorname{degree}(E)+\sum_{x \in S} \operatorname{trace}(A(x))=0 .
$$

In Lemma 2.3, set $L=\bigwedge^{r} E$, where $r=\operatorname{rank}(E)$, and $\lambda_{x}=\operatorname{trace}(A(x))$ for every $x \in S$. Let

$$
\widetilde{\phi}_{L} \in H^{0}(X, \operatorname{End}(L))^{*}=\mathbb{C}^{*}
$$

be the class for $\left(L,\left\{\lambda_{x}\right\}_{x \in S}\right)$ (as in (3.8)). It is straight-forward to check that

$$
\widetilde{\phi}_{E}^{A}\left(\operatorname{Id}_{E}\right)=\widetilde{\phi}_{L}(1)
$$

(see (3.8) for $\left.\widetilde{\phi}_{E}^{A}\right)$. In view of (3.9), from Lemma 2.3 it follows that $L$ has a logarithmic connection with residue $\lambda_{x}$ at every $x \in S$. Therefore, we have $\widetilde{\phi}_{L}(1)=0$. Hence from the above equality it follows that $\widetilde{\phi}_{E}^{A}\left(\operatorname{Id}_{E}\right)=0$. Now we conclude that $\widetilde{\phi}_{E}^{A}=0$, because $E$ is simple.

Since all stable vector bundles are simple, Lemma 3.2 has the following corollary.

Corollary 3.3. Let $E$ be a stable vector bundle. Then the following two statements are equivalent:

(1) There is a logarithmic connection on $E$ singular over $S$ with residue $A(x)$ for every $x \in S$.

(2) The collection of endomorphisms $A(x), x \in S$, satisfy the condition

$$
\operatorname{degree}(E)+\sum_{x \in S} \operatorname{trace}(A(x))=0 .
$$

\section{RIGID ENDOMORPHISMS AND LOGARITHMIC CONNECTIONS ON VECTOR BUNDLES}

4.1. Rigid endomorphisms. The vector space

$$
H^{0}(X, \operatorname{End}(E))=H^{0}\left(X, E \otimes E^{*}\right)
$$

is a Lie algebra for the operation $[v, w]=v \circ w-w \circ v$. For any point $x \in X$, let

$$
s_{x}:=H^{0}(X, \operatorname{End}(E)) \longrightarrow \operatorname{End}\left(E_{x}\right)=\operatorname{End}(E)_{x}, \quad v \longmapsto v(x),
$$

be the evaluation map. Now define the Lie subalgebra

$$
I(x):=\operatorname{image}\left(s_{x}\right) \subset \operatorname{End}\left(E_{x}\right) .
$$

An element $\alpha \in \operatorname{End}\left(E_{x}\right)$ will be called rigid if

$$
[\alpha, I(x)]=0,
$$

meaning $\alpha \circ v(x)=v(x) \circ \alpha$ for all $v \in H^{0}(X, \operatorname{End}(E))$.

If $H^{0}(X, \operatorname{End}(E))=\mathbb{C} \cdot \operatorname{Id}_{E}$, then all elements of $\operatorname{End}\left(E_{x}\right)$ are rigid. 
4.2. Criterion for logarithmic connections on indecomposable bundles. A holomorphic subbundle $F$ of a holomorphic vector bundle $V$ on $X$ is called a direct summand if there is another holomorphic subbundle $F^{\prime}$ of $V$ such that the natural homomorphism

$$
F \oplus F^{\prime} \longrightarrow V
$$

is an isomorphism. A holomorphic vector bundle $V$ is called indecomposable if there is no direct summand $F$ of it such that $0<\operatorname{rank}(F)<\operatorname{rank}(V)$.

As before, $S$ is an effective reduced divisor on $X$, and $E$ is a holomorphic vector bundle on $X$.

Proposition 4.1. Assume that $E$ is indecomposable. For each point $x \in S$, fix a rigid endomorphism $A(x) \in \operatorname{End}\left(E_{x}\right)$. Then the following are equivalent:

(1) There is a logarithmic connection on $E$ singular over $S$ such that the residue is $A(x)$ for every $x \in S$.

(2) The collection $A(x), x \in S$, satisfy the condition

$$
\operatorname{degree}(E)+\sum_{x \in S} \operatorname{trace}(A(x))=0 .
$$

Proof. As noted in the proof of Lemma 3.2, if $E$ admits such a logarithmic connection, then (4.1) holds.

To prove the converse, assume that (4.1) holds. In view of Lemma 2.2, we need to show that the short exact sequence in $(2.7)$ splits, or in other words, $\widetilde{\phi}_{E}^{A}$ in (3.8) is the zero homomorphism.

Since $E$ is indecomposable, all elements of $H^{0}(X, \operatorname{End}(E))$ are of the form $c \cdot \operatorname{Id}_{E}+N$, where $c \in \mathbb{C}$ and $N$ is a nilpotent endomorphism of $E$ [At2, p. 201, Proposition 15]. In the proof of Lemma 3.2 it was shown that (4.1) implies that $\widetilde{\phi}_{E}^{A}\left(\operatorname{Id}_{E}\right)=0$.

Let $N \in H^{0}(X, \operatorname{End}(E))$ be a nilpotent endomorphism. It is known that

$$
\widetilde{\phi}_{E}^{0}(N)=0
$$

[At2, p. 202, Proposition 18(ii)] (see (3.7) for $\widetilde{\phi}_{E}^{0}$ ). Therefore, in view of Proposition 3.1, to prove that $\widetilde{\phi}_{E}^{A}(N)=0$, it suffices to show that

$$
\widetilde{\gamma}_{x}(A(x))(N)=0
$$

for all $x \in S$, where $\widetilde{\gamma}_{x}$ is constructed in (3.5).

Since $N(x) \in \operatorname{End}\left(E_{x}\right)$ is nilpotent, and $A(x)$ commutes with $N(x)$, we conclude that $\operatorname{trace}(A(x) \circ N(x))=0$. In view of this, (4.2) follows from (3.6).

\subsection{Criterion for logarithmic connections with rigid residues. Let}

$$
E=\bigoplus_{i=1}^{n} E^{i}
$$


be a decomposition of $E$ into a direct sum of indecomposable vector bundles. If

$$
E=\bigoplus_{i=1}^{n^{\prime}} F^{i}
$$

is another such decomposition, then $n=n^{\prime}$ and there is permutation $\delta$ of $\{1, \cdots, n\}$ such that $E^{i}$ is holomorphically isomorphic to $F^{\delta(i)}$ [At1, p. 315, Theorem 2]. It is known that such a decomposition is obtained by choosing a maximal torus in the algebraic group $\operatorname{Aut}(E)$ consisting of the holomorphic automorphisms of $E$ [At1]. Let

$$
\mathcal{T}=\left(\mathbb{G}_{m}\right)^{n} \subset \operatorname{Aut}(E)
$$

be the maximal torus corresponding to the decomposition in (4.3), where $\mathbb{G}_{m}=\mathbb{C} \backslash\{0\}$ is the multiplicative group. So the action of any $\left(\mu_{1}, \cdots, \mu_{n}\right) \in\left(\mathbb{G}_{m}\right)^{n}$ on $E$ sends any $\left(w_{1}, \cdots, w_{n}\right) \in \bigoplus_{i=1}^{n} E^{i}$ to $\left(\mu_{1} w_{1}, \cdots, \mu_{n} w_{n}\right)$.

For each point $x \in S$, fix a rigid endomorphism $A(x) \in \operatorname{End}\left(E_{x}\right)$. Since $A(x)$ commutes with $\mathcal{T}$, it follows that

$$
A(x)\left(E_{x}^{i}\right) \subset E_{x}^{i}
$$

for all $1 \leq i \leq n$ and all $x \in S$. The endomorphism

$$
\left.A(x)\right|_{E_{x}^{i}}: E_{x}^{i} \longrightarrow E_{x}^{i}
$$

will be denoted by $A_{x}^{i}$.

If $D: E \longrightarrow E \otimes K_{X} \otimes \mathcal{O}_{X}(S)$ is a logarithmic connection on $E$ singular over $S$ with residue $A(x)$ at every $x \in S$, then the composition

$$
E^{i} \hookrightarrow E \stackrel{D}{\longrightarrow} E \otimes K_{X} \otimes \mathcal{O}_{X}(S) \stackrel{q^{i} \otimes I d}{\longrightarrow} E^{i} \otimes K_{X} \otimes \mathcal{O}_{X}(S)
$$

where $q^{i}: E \longrightarrow E^{i}$ is the natural projection obtained from (4.3), is a logarithmic connection on $E^{i}$ singular over $S$ with residue $A_{x}^{i}$ at every $x \in S$. Conversely, if for every $1 \leq i \leq n$

$$
D^{i}: E^{i} \longrightarrow E^{i} \otimes K_{X} \otimes \mathcal{O}_{X}(S)
$$

is a logarithmic connection on $E^{i} \subset \bigoplus_{i=1}^{n} E^{i}$ singular over $S$ with residue $A_{x}^{i}$ at every $x \in S$, then $\bigoplus_{i=1}^{n} D^{i}$ is a logarithmic connection on $E$ singular over $S$ with residue $A(x)$ at every $x \in S$.

Note that every direct summand of $E$ is a part of a decomposition of $E$ into a direct sum of indecomposable vector bundles. Now using Proposition 4.1 we have the following:

Theorem 4.2. For each point $x \in S$, fix a rigid endomorphism $A(x) \in \operatorname{End}\left(E_{x}\right)$. Then the following hold:

(1) For every direct summand $F \subset E$,

$$
A(x)\left(F_{x}\right) \subset F_{x}
$$

for every $x \in S$. 
(2) The vector bundle $E$ admits a logarithmic connection on $E$ singular over $S$, with residue $A(x)$ at every $x \in S$, if and only if for every direct summand $F \subset E$,

$$
\operatorname{degree}(F)+\sum_{x \in S} \operatorname{trace}\left(\left.A(x)\right|_{F_{x}}\right)=0 .
$$

\section{ACKNOWLEDGEMENTS}

We thank the referee for helpful comments. The first-named author is supported by a J. C. Bose Fellowship.

\section{REFERENCES}

[At1] M. F. Atiyah, On the Krull-Schmidt theorem with application to sheaves, Bull. Soc. Math. Fr. 84 (1956), 307-317.

[At2] M. F. Atiyah, Complex analytic connections in fibre bundles, Trans. Amer. Math. Soc. 85 (1957), 181-207.

[De] P. Deligne, Equations différentielles à points singuliers réguliers, Lecture Notes in Mathematics, Vol. 163, Springer-Verlag, Berlin-New York, 1970.

[GH] P. Griffiths and J. Harris, Principles of algebraic geometry, Pure and Applied Mathematics. Wiley-Interscience, New York, 1978.

[La] S. Lang, Algebra, Revised third edition, Graduate Texts in Mathematics, 211, Springer-Verlag, New York, 2002.

[Oh] M. Ohtsuki, A residue formula for Chern classes associated with logarithmic connections, Tokyo Jour. Math. 5 (1982), 13-21.

[We] A. Weil, Généralisation des fonctions abéliennes, Jour. Math. Pure Appl. 17 (1938), 47-87.

School of Mathematics, Tata Institute of Fundamental Research, Homi Bhabha Road, MUMBAi 400005, INDIA

E-mail address: indranil@math.tifr.res.in

BCAM - Basque Centre for Applied Mathematics, Alameda de Mazarredo 14, 48009 Bilbao, SPAin

E-mail address: adan@bcamath.org

School of Mathematics, Tata Institute of Fundamental Research, Homi Bhabha Road, Mumbai 400005, IndiA

E-mail address: apmath90@math.tifr.res.in 